\title{
Kommentar zu einem Kommentar über „Inter mirifica“
}

\author{
von Karl Höller
}

Als einen "Mantel barmherzigen Schweigens" interpretiert Otto Karrer ${ }^{1}$ die in der bisherigen Konzilsliteratur relativ stiefmütterliche Behandlung des Dekrets "Inter mirifica" über die sozialen Kommunikationsmittel. Was kleinere Kommentarwerke ${ }^{2}$ stillschweigend übergingen, konnte jedoch in der bislang umfassendsten postkonziliaren deutschen Nachlese nicht unberücksichtigt bleiben: Im ersten Teil des dreibändigen Ergänzungswerks zum Lexikon für Theologie und Kirche, „Das Zweite Vatikanische Konzil, Dokumente und Kommentare", wird auch das Publizistik-Dekret ${ }^{3}$ mit den Methoden eines "wissenschaftlichen Kommentars" - wie ihn die Einführung ins Gesamtwerk ankündigt - exegetisiert ${ }^{4}$. Doch zwischen dem Streben nach Wissenschaftlichkeit und der Verwirklichung ihrer Anforderungen versiegt diese Beurteilung von "Inter mirifica " auf der Durststrecke einer begrenzten Sachkenntnis und unzulänglicher Argumentation. Zum viel kritisierten Konzilsdekret gesellt sich eine nicht weniger kritisierbare Deutung, die sich in einem theologischen Standardwerk, das auf Jahre hin in den Bibliotheken der Seelsorger zu finden sein wird, "nur nachteilig für die nachkonziliare Arbeit auf diesem Gebiet gesellschaftlicher Kommunikation auswirken wird", wie eine erste Rezension ${ }^{5}$ bemerkt.

Einer sozusagen halboffiziellen, unter dem Protektorat eines Kardinals und eines Erzbischofs erstellten Konzils-Auslegung kommt nach fünfjährigem Warten auf die entsprechende Pastoralinstruktion und den fortwährenden Schwierigkeiten ${ }^{6}$ der Päpstlichen Kommission bei der Abfassung eben dieser Ausführungsbestimmungen 
erhebliche Bedeutung zu. Die folgende Auseinandersetzung mit dem Kommentar soll - soweit dies möglich ist - eine Multiplikation seiner Fehler und Schwächen verhindern, ohne dabei eine neue Deutung von "Inter mirifica“ geben zu wollen. Sie folgt der Gliederung des Kommentars, an dessen Einführung in die Vor- und Entstehungsgeschichte des Konzilsdokuments (S. 112-115) sich auf den Seiten 116 bis 135 der lateinische (nach den AAS $^{7}$ ) und der deutsche ${ }^{8}$ Text des Dekrets mit fortlaufenden Erklärungen am Fuß der Seiten anschließen.

\section{Aus zweiter Hand}

Wissenschaftliche Attitüde hat gemeinhin das Bestreben, dem Leser durch eine Fülle von Belegstellen die Möglichkeit zu vertiefender Kenntnisnahme zu geben. Auf den dreieinhalb Seiten der Einführung sucht man - wie im gesamten Kommentar vergeblich nach Quellen- und Literaturangaben. Nur auf Seite 120 deutet sich ein einziges Mal mit dem in Klammern gesetzten Namen des Münchener Zeitungswissenschaftlers Otto B. Roegele hinter einem angeführten Nebensatz korrektes Zitieren an. Doch dem kundigen Leser bleibt schon nach wenigen Sätzen der Einleitung nicht verborgen, daß hier des gleichen Roegele verdienstvolle erste Würdigung des Dekrets ${ }^{\theta}$ allenthalben, zuweilen bis ins Detail, Hebammendienste geleistet hat. Was Roegele mit 54 Fußnoten absicherte und dennoch als „vorläufige Information" verstanden wissen wollte, "die noch in mehrfacher Hinsicht der Ergänzung, vielleicht auch der Korrektur bedarf " 10 , wird grob gerastert und undifferenziertapodiktisch vorgetragen. Von einer Vorsicht, „die um die Mehrschichtigkeit der Wirklichkeit weiß, [ist] nichts mehr zu spüren."11

Nach leichten Retuschen greift der Autor auch auf das Vokabular seines - soweit ersichtlich - einzigen Gewährsmannes zurück. Wurde dem vorbereitenden Sekretariat - nach Roegele (S. 306) - ,eine Reihe von Fragepunkten vorgelegt, die eine erste Orientierung für seine Arbeit abgeben sollten“, so lautet dieser Sachverhalt in der Fassung unseres Kommentars (S. 112): „Dem Sekretariat wurden zu Beginn seiner Arbeit eine Reihe Fragen vorgelegt, an denen es sich orientieren sollte." Fast deckungsgleich sind auch die Sätze über den Inhalt dieser Fragen, mit dem einen, charakteristischen Unterschied, daß Roegele sie durch ein vorsichtiges "Soweit bekannt" einleitet. Werden im Original (S. 308) Gedanken der Konzilsväter "ganz offen", dann in der Ubernahme (S. 112) "ziemlich unverblümt" ausgesprochen. Die Liste solcher Gegenüberstellungen ließe sich beliebig fortsetzen. ${ }^{12}$

Bei soviel Kongruenz wecken die Abweichungen von der Regel besonderes Interesse. So hieß das vorbereitende Sekretariat für die sozialen Kommunikationsmittel im Italienischen nicht „Segretariato della stampa e dello spettacolo“, sondern „Segretariato per la stampa e lo spettacolo"13, und sein Sekretär nicht Deskurs, sondern Deskur. In der Reihenfolge, wie die deutschen Beteiligten genannt werden, gehörten sie nur dem vorbereitenden Sekretariat an. ${ }^{14}$ Der Autor übergeht dessen Auflösung nach der Vorbereitungsphase und die Ubernahme der Arbeiten in die Kommission für das Laienapostolat, zu der von deutscher Seite der Essener Bischof Franz Hengsbach als Mitglied, sowie P. Hirschmann SJ und später (1963) Msgr. Siegel als Periti zählten.

Aus der Rede des Erzbischofs Stourm von Sens in der Konzilsaula zitiert der Autor die relativ unwesentlichen und zudem ungeprüften Zahlen über die Verbreitung der modernen Massenmedien in der Welt, auf die Roegele verzichtet, um 
Wesentlicheres hervorzuheben: daß nämlich Erzbischof Stourm als der von der Kommission bestellte Berichterstatter (relator) fungierte. Ein Vergleich mit der reportierenden Konzilsliteratur ${ }^{15}$ weckt $Z$ Weifel, ob die hier vorgelegten Resümees der Väter-Interventionen überhaupt deren entscheidenden Inhalt wiedergeben. „Eine Darstellung und Wertung der Diskussion in der Aula ist ohne genaue Kenntnis der einzelnen Beiträge nicht zu erstellen."16 Falls dem Autor, der 1965 zum Konsultor der postkonziliaren Päpstlichen Kommission für die publizistischen Mittel ernannt wurde ${ }^{17}$, die Väter-Texte im Wortlaut nicht zugänglich waren, blieb er immerhin den Hinweis auf das Subjektive seiner eklektizistischen Methode schuldig. Ferner hätte man sich vom vorliegenden Kommentar auch einige Aufschlüsse über die Arbeitsdiskussion innerhalb des vorbereitenden Sekretariates und der späteren Konzilskommission gewünscht. Unverhältnismäßig breiten Raum - eine ganze Seite von dreieinhalb Seiten der Einleitung - widmet der Autor dagegen den späten Interventionsmanövern zu Ende der zweiten Sitzungsperiode, wobei er eine Gruppe amerikanischer Konzilsjournalisten als „Fachleute" apostrophiert und ihre FlugblattEinwände, die Roegele im Anhang (S. 344) dokumentiert, kommentarlos vorträgt. Er nennt in diesem Zusammenhang nur drei angesehene Konzilstheologen als Tutoren des Protest-Papiers, obwohl Roegele (S. 312) als auch Xavier Rynne ${ }^{18}$ noch den deutschen Redemptoristen Prof. Dr. Bernhard Häring anführen.

\section{Nicht auf der Höbe der Diskussion}

Im letzten Absatz seiner Einleitung faßt der Autor auf S. 115 zusammen, was nach solcher Vorarbeit vom Konzilsdekret über die sozialen Kommunikationsmittel zu halten sei. Ihm genügen 23 Zeilen für eine pauschale Ablehnung, die mithin vorwegnimmt, was eigentlich den nachfolgenden Textkommentar zu beschließen hätte. Man braucht dem Dekret nicht ausschließlich wohlwollend gegenüberzustehen, um ein solches Verfahren unsachlich-einseitig19 $\mathrm{zu}$ finden. Roegele widmete den „positiven Aspekten" des Konzilsdokuments noch ein eigenes Kapitel20: seiner bloßen Existenz als „Unternehmen ohne Vorbild“21 mit dem Ziel, alle Masse ${ }^{-}$ medien geschlossen in das Feld der Seelsorge einzubeziehen ${ }^{22}$; seiner Offenheit für die weiterführende Diskussion; seinem naiven Optimismus und seinem Verzicht auf kulturkritische Melodramatik.

Man kann das Dekret einen "Lückenbüßer“ schelten, seine frühe Beratung und Verabschiedung in der Aula beklagen, seinen Charakter infolgedessen als präkonziliar bezeichnen, - man darf dann aber in einem postkonziliaren Kommentar nicht den Hinweis auf die ergänzende Thematik in später promulgierten Konstitutionen und Dekreten unterlassen.23 Der Baptist Stanley $\mathrm{I}$. Stuber hat darauf aufmerksam gemacht, "welche chemische Reaktion - oder theologische und pastorale - eintreten würde, wenn man das Dekret über die Werkzeuge der sozialen Kommunikation in die Dogmatische Konstitution über die Kirche und die Pastoralkonstitution über die Kirche in der modernen Welt eintauchte". ${ }^{24}$

Ebenso vermißt man eine geschichtliche Einordnung des Dekrets in die - wenn auch magere, so doch vorhandene - Lehrentwicklung der Kirche zu publizistischen Fragen, etwa zwischen der Enzyklika "Vigilanti cura" vom 29. 6. 1936 und dem Apostolischen Schreiben "Boni Pastoris" vom 22. 2. 1959.25

Unbeeindruckt von solchen Lücken im eigenen Elaborat mündet die gesamte Einleitung des Kommentars in die Klage, das Dekret stehe "weder auf der Höhe der 
konziliären noch auf der der allgemeinen wissenschaftlichen Diskussion." Es kann nicht am Platzmangel gelegen haben, daß eine nähere Erklärung dieses Vorwurfs fehlt; denn eine halbe Seite blieb unbedruckt. Andererseits mußte dem Autor bewußt werden, daß eine solche Behauptung die Ansprüche an die Wissenschaftlichkeit der eigenen Terminologie erheblich hinaufschrauben würde.

\section{Revidiert vom Kommentator}

Der Kommentar bringt die Übersetzung des Konzilsdekrets in der von den deutschen Bischöfen autorisierten und u. a. in der "Herder-Korrespondenz" publizierten Fassung26, „revidiert vom Kommentator". Die angezeigte Revision bezieht sich soweit ersichtlich ${ }^{27}$ - auf eine stilistische Anderung in Art. 11 und die Neufassung des Titels, der, in einer Fußnote der $\mathrm{HK}$ ausführlich begründet ${ }^{28}$, „Dekret über die publizistischen Mittel" hieß. Der Revisor bleibt eine Erklärung seines in mancher Hinsicht zutreffenderen Begriffs „soziale Kommunikationsmittel“ schuldig29, wobei die umfangreichen Überlegungen der konziliaren Vorbereitungskommission in eine weiterführende Deutung hätten eingebracht werden können. ${ }^{30}$ Stehengeblieben ist ein Sinnfehler in Art. 11, wo communicatio mit „Kommunikationsmittel“ übersetzt wird.

Roegele hatte seine Fassung des deutschen Konzilstextes (S. 338-343) stilistisch und sachlich wesentlich verbessert. Diese Verbesserungen hätten sich zur Ubernahme angeboten. Es sei hier nur auf die Artikel 3, 4, 7, 9, 11, 12, 14, 15 und 16 hingewiesen, ohne im einzelnen gegenüberzustellen. ${ }^{31}$ - Wie sehr eine Ubersetzung qualifizierend kommentieren kann, wird aus folgendem Beispiel ersichtlich, das für viele stehen könnte: Art. 3 des Dekrets spricht von der Pflicht der Kirche, mit Hilfe der sozialen Kommunikationsmittel zu verkündigen, „hominesque de eorum recto usu docere". Roegele versteht darunter die pastorale, auf das ewige Heil des Menschen bezogene Aufgabe, „die Menschen über deren richtigen Gebrauch zu belehren“. Unser Kommentar übersetzt: „Grundsätze über deren richtige Anwendung aufzustellen", ein Anspruch, der in solcher Formulierung durchaus als hierarchische Dominanz über publizistische Sachgesetzlichkeit mißdeutet werden kann.31a

\section{Kommentar nach freier Wabl}

Die nachfolgende Beschäftigung mit den am Fuß der lateinisch-deutschen TextDokumentation fortlaufenden exegetischen Deutungen paßt sich der eklektischen Willkür des Kommentars an und bleibt daher weitgehend ungegliedert.

Schon die Auslegung des Vorworts verrät die Interpretations-Perspektive des Kommentars. Als Hauptmotive des Dekrets nennt er die Sorge vor möglichem Mißbrauch der Medien und deren Nutzung für die Evangelisation, während die bischöflichen Verfasser des Dokuments sich darüber hinaus der Hilfen für den Menschen selbst wie der Notwendigkeit einer rechten Förderung der Medien bewußt sind. Ebenso willkürlich wird der Kreis der vom Konzil angesprochenen Adressaten unter die Ägide "amtlicher kirchlicher Kräfte" gestellt.

Statt die Motive des Dekrets zu verzerren, wäre es Aufgabe des Kommentars gewesen, nach entscheidenderen, im Text nicht angesprochenen Gründen für eine Behandlung dieser Dinge im kirchlichen Raum zu fragen. Kann es der Ecclesia 
genügen, pastorale Verhaltensmuster für den Umgang mit den Massenmedien zu liefern, ihre Gefahrenpunkte zu markieren und ihren Nutzwert für das Apostolat zu beschreiben? Stellt sich nicht vorrangig die Aufgabe, weniger fixiert beim Funktionsablauf der medialen Vermittlung des gesellschaftlichen Zwiegesprächs durch instrumentierte Kanäle zu verweilen, als ein gelösteres Verständnis aus der analogen Deutung innertrinitarischen Lebens, „das sich im Bereich der Mitteilung vollzieht" ${ }^{\text {32, }}$ zu gewinnen? Werden nicht durch die Inkarnation Christi Mensch und Schöpfung in diese göttliche Kommunikation einbezogen? Erhalten nicht irdische Worte und Zeichen durch den Logos neue Dimensionen? Ist nicht die Kirche „bleibende Präsenz des endgültigen und darum reflex ausdrïcklichen Wortes Gottes an die Welt, in der Welt und für die Welt"?33 Kann sich nicht in kirchlicher Gemeinschaft und Verkündigung Kommunikation zu personaler Kommunion verdichten ${ }^{34}$ - Was nützt es, das Fehlen aller Arten von Theologien (des Bildes, der Kommunikation etc.) zu beklagen, mögliche Ansätze aber nicht einmal anzudeuten? Auch von Seiten der publizistischen Wissenschaft ist der Brückenschlag längst begonnen, ohne daß davon sonderlich Notiz genommen wird. ${ }^{35}$

Was sich bereits in der UUbersetzung des Art. 3 (siehe oben) andeutete, bestätigt die Art der Kommentierung: Der Autor bemerkt ein Übergewicht des kirchlichen Rechtsanspruchs auf Besitz und Benutzung der Kommunikationsmittel, wo durchaus auch von der Pflicht die Rede ist, mit ihrer Hilfe die Heilsbotschaft zu verkündigen. Ebensowenig bezieht sich die den Oberhirten aufgetragene institutio und moderatio auf die Mittel, sondern auf das Ziel, Heil und Vollendung zu erstreben, ein Auftrag, "der offen ist für die verschiedenen Grade der Partizipation durch andere ". ${ }^{\mathbf{3 6}}$ Immer wieder hakt der Kommentar seine Kritik an Worten und Satzgruppen des Dekrets ein, die soeben noch ausreichende Angriffsflächen bieten, um beckmessernd zu rechten. Daß z. B. die Aufgabe der Laien im Text mit einem "ceterum" eingeleitet wird, berechtigt nun wirklich nicht zu der Schlußfolgerung, dem ganzen Dokument eigne eine klerikalistische Grundtendenz. Solche Detail-Querelen verstellen den Blick auf die grundlegenderen Schwächen der Vorlage, die - wie sich zeigen wird - auch die Schwächen des Kommentars sind. Beide scheitern daran, publizistisch-kommunikative Vorgänge mit dem Instrumentarium der philosophischen Ethik messen zu müssen. Beide bleiben insofern zeitungswissenschaftlich-vorkonziliar, als sie zu einseitig auf die Wirkung publizistischer Aussagen in der Gesellschaft fixiert sind, den publizistischen Prozeß als Monolog begreifen, gleichsam als dirigistischen Einbahnverkehr vom Aussageträger zum Aussageempfänger. ${ }^{37}$

Dem widerspricht nicht die Bemerkung Roegeles ${ }^{38}$, der Titel des Dekrets berühre "Vegetationsspitzen von Forschung und Lehre“. Er allein ist neueren Theorien über den Kommunikationsvorgang entlehnt, der grundsätzlich als ein interdependentes Wechselspiel zwischen Publizist und Publikum gesehen wird, als ständiger Gesprächs-Kreislauf, in dem die Rezipienten durch Inspiration des Kommunikators aktiv werden, als ein horizontal verstandener Rückkoppelungs-Vorgang, der schon zur freilich anfechtbaren Bezeichnung der Publizistik-Wissenschaft als "Regelungslehre" geführt hat. ${ }^{39}$

Weil solche Ansätze dem Dekret fremd waren, hätte man sie sich vom Kommentar erwähnt gewünscht. Funktional-dialogische Sicht hätte die Beurteilung von "Inter mirifica " entkrampft. Sie hätte nicht die Aufgaben von Priestern und Laien im Bereich der publizistischen Mittel als gegeneinander konkurrierende Einflußnahmen herausgearbeitet, sondern ihr Miteinander in Kommunikator- und Rezipientenfunktion innerhalb der Kirche als dem "Volk Gottes " betont. Funktional-dialogische 
Betrachtung würde auch nichts aus Art. 4 des Dekrets „Ausgangspunkte zu einer Phänomenologie der sinnlichen Wahrnehmung" herauslesen, wo es um sittlichmoralische Gesichtspunkte geht, noch die Furcht der Väter vor der Wirkungsart mancher Medien mit der Behauptung übersteigern, „daß der Mensch dem übermittelten Eindruck ausgeliefert ist, ohne sich seiner erwehren zu können." Ihr wäre der Plural „öffentliche Meinungen“, wie ihn das Konzilsdokument gebraucht, weniger Anlaß zu ausgiebiger Polemik als vielmehr dankbarer Anstoß zur Entmythologisierung des überforderten Begriffs ${ }^{40}$ gewesen.

\section{Grundformen und Grundbegriffe der Kommunikation}

Der Autor vermißt im Dekret mit Recht eine theologisch-philosophische Reflexion über Wesen und Eigenart der technisch vermittelten Kommunikation. Wo er jedoch versucht, die schon vorhandenen soziologisch-sozialpsychologischen Vorarbeiten zu erläutern, blockiert eine popularwissenschaftliche Terminologie den Zugang. So spricht er z. B. (S. 122) von den „Darbietungen“ der Kommunikationsmittel, die man unter den beiden Aspekten der Information und des Schauspiels betrachten könne. Die Medien sind jedoch „Träger öffentlicher Aussagen“, die sie als Berichterstattung (Information), Meinungsbildung (Kommentar) und Unterhaltung vermitteln, wobei das Schauspiel (Theater) selbst als Medium fungieren kann.

Als wenig ergiebig erweist sich auch der verhältnismäßig breit angelegte Exkurs (S. $119 \mathrm{ff}$.) über Grundformen und Grundbegriffe der Kommunikation. Einseitig wird die Information als „der wesentliche Inhalt der durch die technischen Mittel vermittelten Kommunikation" genannt. Hinweise auf Kommentator- und Partnerfunktion unterbleiben. Information tritt nicht - wie der Kommentar überschätzend meint - „an die Stelle der Wirklichkeit“. Sie kann im Gegenteil inkongruent auch als Unterhaltung rezipiert werden. Die pessimistische Beurteilung von Konzentrationsbestrebungen als einer Hauptgefahr auf dem Gebiet der publizistischen Mittel verrät den Anhänger einsinnig ausgerichteter "Weisungslehre“. Wo bleibt an dieser Stelle ein Wort über die Rangordnung (Hierarchie) ${ }^{41}$ der Medien? Der Autor wünscht sich (S. 120) vom Konzil einen Hinweis auf den Solidaritätseffekt ${ }^{42}$ weltweiter Information und zitiert in diesem Zusammenhang zum ersten und einzigen Mal Otto B. Roegele - allerdings ohne genauen Fundstellenhinweis ${ }^{43}$ übersieht jedoch dabei, daß sein Gewährsmann gerade mit diesem entliehenen Zitat „positive Aspekte des Dekrets“ beleuchtet.

\section{Vom Recht auf Information}

Der Kommentar erkennt das besondere Gewicht des Art. 5 über das Recht des Menschen auf Information. Leider fehlt eine Erläuterung der im Dekret angesprochenen „ethischen Grundsätze“, die „beim Sammeln und Verbreiten von Nachrichten" beachtet werden müssen. Man hätte sich eine solche Erläuterung gewünscht, $\mathrm{da}$ in der kirchlichen Bürokratie gerne als deontologisches Peccatum des Journalistenberufs geächtet wird, was als schlichte Information gedacht war, aber kirchenpolitisches oder pastorales Taktieren durchkreuzt. Hier könnte des Autors berechtigte Frage nach den fehlenden Außerungen über Meinungsfreiheit im innerkirchlichen Raum anschließen. Er widmet diesem Thema den gebührenden Raum, zitiert Pius 
XII. als wichtigsten Kronzeugen, belegt aber wiederum nicht seine Funde. ${ }^{44}$ Auch ist es nicht korrekt, dem Dokument das Ubergehen eines so wichtigen Themas mit dem Hinweis auf die ansonsten enge Anlehnung an die Pius-Enzyklika „Miranda prorsus" anzukreiden. In dieses Rundschreiben hat der Papst die 1950 geäußerten Gedanken mit keiner Andeutung aufgenommen. ${ }^{45}$ Vielmehr hätte der Kommentator an das Konzil die Frage richten können, ob die Väter das genannte Problem bei ihren sonstigen Diskussionen anschnitten. Sie übergingen es nämlich nicht. In der Sitzung vom 22. Oktober 1963, als über das Verhältnis der Laien zu den „Hirten der Kirche“ gesprochen wurde, betonte der Spanier Enrique y Taracón die Notwendigkeit freier Meinungsäußerung in der Kirche, die kommen und sichergestellt werden müsse. Fehle es daran, falle die Schuld auf die Bischöfe. ${ }^{\mathbf{4 6}}$

\section{Freibeit der Kunst und Darstellung des Bösen}

Von einem Kommentar erwartet der Leser Antworten auf Fragen, die sich bei der Lektüre stellen und nicht die indirekte Wiedergabe des zu kommentierenden Textes. Gerade weil das Konzil sich bei der Behandlung so diffiziler Probleme wie „Freiheit der Kunst" und "Darstellung des sittlich Bösen“ in ungewohnten Grenzgebieten der Moraltheologie bewegen mußte, wo nur der Kompaß der scholastischen Naturrechtslehre verläßliche Orientierung bot, hätte im Nachhinein auf die Mängel solcher Verfahrensweise verwiesen werden müssen ${ }^{47}$ : Wo konkret kann die Kunst gegen das alle anderen Gesetze umgreifende Sittengesetz verstoßen? Ist es wirklich der wesentliche Sinn künstlerischen Schaffens, die Wahrheit der Welt auszudrücken? Wie unterscheidet sie sich bei solcher Definition von der Wissenschaft? Welche Themen gebieten bei der Darstellung des sittlich Bösen eine "gewisse Zurückhaltung", wecken im Menschen "leicht niedere Instinkte" (welcher Art?)? Wie verteilt die Moraltheologie Verantwortlichkeit und beurteilt sittliches Fehlverhalten, wenn der Erfolg publizistischen Funktionierens, den Kommunikator und Rezipienten gleichermaßen effektuieren, nach der "angenehmsten Bedürfnisbefriedigung " 48 gemessen wird? Letztere Frage eröffnet eine Perspektive, nach der auch die sich anschließenden Artikel 9, 10 und 11 des Dekrets über die Pflichten „der aktiv oder passiv an den Kornmunikationsmitteln Beteiligten“ hätten kommentiert werden können.

\section{Eingriffe der öffentlichen Gewalt}

Den im bedeutsamen Art. 12 des Konzilsdokuments aufgeführten staatlichen Pflichtenkatalog zum Schutz der Sitten und des gesellschaftlichen Fortschritts nennt der Kommentar ,vorsichtig und abgewogen". Er ist es in der Tat. Man wird nicht gleich die "Besonnenheit“ der Konzilsväter in Abrede stellen, wenn man trotzdem bei der Text-Interpretation auf die Gefahrenquelle hinweist, die jedwede Delegierung von Rechten an anonyme Größen in sich birgt. Wer definiert, was nach mißliebigem publizistischem Handeln zur Verantwortung zu ziehen ist? Wer reguliert das Niveau des gesellschaftlichen Wertkodex, bestimmt in einer pluralistischen Gesellschaft voll divergierender „Güter“ die Qualität des vorrangig zu schützenden Gutes? Ohne Antwort auf diese Fragen bleiben die konziliaren Weisungen gefährlich inkonkret. Man braucht dabei nicht gleich Gefahren zu beschwören, wie sie der 
Konzilskommentator Mac Eoin sieht ${ }^{40}$ : „Es [das Schema, d. Verf.] stellt Prinzipien auf, ... die in der Tat auch den kommunistischen Staaten recht geben, wenn sie religiöse Veröffentlichungen verbieten.“

\section{Prelum bonestum}

Das zweite Kapitel des Dekrets über den Einsatz der Kommunikationsmittel im Dienst des Apostolates beginnt in Art. $13 \mathrm{mit}$ der Aufgabenverteilung. Uber dem Ärer, daß die Laien wieder einmal zu kurz kommen, entgeht dem Kommentator die Schwäche des eigentlichen Ansatzes, der leider nicht, wie Roegele bemerkt ${ }^{50}$, vom Begriff des Zeugnisgebens, sondern von der „durchaus traditionellen Sicht intentionaler Beeinflussung" ausgeht.

Der gelungenen Deutung (S. 128 f.) des Art. 14, der sich mit dem Medium Presse beschäftigt, kann man zustimmen. Diese Zustimmung wird ein wenig gemindert durch die vertane Chance, an dieser Stelle endgültig und unwiderruflich den für das Verhältnis von Kirche und Publizistik seit mehr als hundert Jahren so verhängnisvollen Begriffs der "guten Presse" verabschiedet zu haben.51 Daß er Eingang ins Konzilsdekret fand, zeugt von der Hartnäckigkeit seiner Existenz. Er spiegelt die grundlegende Misere katholischer Pressepolitik. Moralische Wertbegriffe gängeln publizistische Sachgesetze: „Gut“ ist die ethische Forderungen respektierende und sie unterstützende, die der rechten Staatslehre dienende, die ordnende, wegweisende, in jedem Sinn wohlwollend-wohlgesinnte, nicht vor dem Publikumsgeschmack buckelnde und darum als Opfer ihrer Anständigkeit selten erfolgreiche Presse. Wenn irgendwo, dann wäre hier ein Nachruf auf dieses unheilvolle Konglomerat fällig gewesen.

Bei der Behandlung des Mediums Film (S. 129) fehlt ein Hinweis auf die Problematik kirchlicher Preisverleihung und Bewertung. ${ }^{52}$ - Wenn die katholischen Rundfunkstationen aufgezählt werden, darf der kostspielige Aufbau des Senders Manila nicht ungenannt bleiben. - Die beiläufige Erwähnung des Theaters im Konzilsdekret hätte den Kommentator nicht von einer Würdigung seiner medialen Funktion $^{53}$ abhalten sollen (S. 131). - Zu unvollständig ist die Aufzählung katholischer Organisationen auf dem Gebiet der Kommunikationsmittel. Selbst die letzten Zeilen des Kommentars bedürfen der Korrektur: Die internationale katholische Dachorganisation der Presse hat nach ihrem 7. Weltkongreß 1965 in New York den Namen geändert und heißt heute "Union Catholique Internationale de la Presse“ (UCIP).

\section{Der Bouillonwürfel}

Msgr. Anton Kochs, Leiter der Katholischen Filmarbeit in Deutschland, hat in Referaten $^{54}$ über "Inter mirifica" wiederholt die Metapher vom Bouillonwürfel auf das Dekret angewandt: Es sei ein Konzentrat sorgfältiger vorbereitender Arbeiten, das erst nach Aufkochen genießbar werde. Bleibt man im Urteil über den vorliegenden Kommentar bei dieser Metapher, wird man feststellen müssen, daß die Menge des beigegebenen Wassers über die Schmackhaftigkeit der Bouillon entscheidet. Von der längst überfälligen Pastoralinstruktion bleibt zu hoffen, daß sie nicht den Erwartungen des Kommentators entspricht und „alle in dem Dekret angeordneten Maßnahmen" noch einmal zusammenfaßt. Es wäre schade um die Bouillon. 


\section{Anmerkungen:}

1. Otto Karrer: Das Zweite Vatikanische Konzil. Reflexionen zu seiner geschichtlichen und geistlichen Wirklichkeit, München 1966, S. 211.

2. Vgl. etwa: Zweites Vatikanisches Okumenisches Konzil, Dokumente mit den authentischen lateinischen Texten der AAS und der Ubersetzung im Auftrag der Deutschen Bischöfe, Münster 1965-1968, 15 Hefte, „eingeleitet von Konzilsvätern, Konzilsberatern und Gelehrten".

3. Karlheinz Schmidthüs: Dekret über die sozialen Kommunikationsmittel, in: „Lexikon für Theologie und Kirche": Das Zweite Vatikanische Konzil. Konstitutionen, Dekrete, Erklärungen, Teil I (Hrsg.: Heinrich Suso Brechter, Bernhard Häring, Josef Höfer u. a. m., Schriftleitung Herbert Vorgrimler), Freiburg 1966, S. 111-135.

4. Ebd.: Herbert Vorgrimler: Zur Einführung, S. 7.

5. Kirchliche Hauptstellen der Katholischen Rundfunk- und Fernseharbeit sowie die Kirchliche Hauptstelle für Bild- und Filmarbeit e. V. (Hrsg.): Kritische Bemerkungen zu dem Kommentar betr. Konzilsdekret "Inter mirifica“ im Ergänzungsband des Lexikons für Theologie und Kirche, hektografiert, $17 \mathrm{~S}$. - Auf diese sehr sorgfältig und kenntnisreich ausgearbeiteten "Kritischen Bemerkungen" wird im ff. (zitiert als KB) mehrmals verwiesen. Leider sind sie nur bedingt als objektive Auseinandersetzung mit dem Herder-Kommentar zu betrachten, da sie zum Teil apologetischen Eifer verraten, aus dem die Rechtfertigung namhafter Vertreter der Kirchlichen Hauptstellen herausklingt, die an der vorbereitenden Konzeption des späteren Konzilsdekrets beteiligt waren.

6. Siche: Instruktions-Entwurf zum Publizistik-Dekret abgelehnt, in: „Communicatio Socialis", 1:1968, Heft 4, S. 355.

7. Acta Apostolicae Sedis, $56: 1964$, S. $145-157$.

8. Deutsche Ubersetzung, besorgt in Auftrag der deutschen Bischöfe von der Publizistischen Kommission der Deutschen Bischofskonferenz (siehe Anm. 26), revidiert vom Kommentator.

9. Otto B. Roegele: Das Konzilsdekret „Uber die Werkzeuge der sozialen Kommunikation“, in: „Publizistik“, Bremen, 9:1964, S. 305-347.

10. Roegele, a.a.O., S 305.

11. KB, a.a.O., Nr. 2, S. 1.

12. So mit Roegele (S. 308) und Komm. (S. 112), wo davon die Rede ist, daß nach Erörterung des schwierigen Offenbarungsschemas das Bedürfnis bestand, sich einem leichteren Stoff zuzuwenden. - Roegele (S. 310) und Komm. (S. 113) über den Abschluß der Konzilsdebatte in der ersten Sitzungsperiode. - Roegele (S. 311) und Komm. (S. 113): Nach zwei Sitzungsperioden wollte man nicht mit nur einem fertigen Dokument vor die Offentlichkeit treten. Roegele versucht, diese Vermutung begründend zu stützen (Fußnote 24, S. 331), Komm. behauptet. - Wie Roegele spricht auch Komm. von Dr. Karl August Siegel, statt Msgr. Karl August Siegel, - damals Leiter der Bischöflichen Hauptstelle (nicht: Hauptarbeitsstelle, wie Komm. schreibt) für Katholische Fernseharbeit in Deutschland.

13. Vgl.: Il Concilio Ecumenico Vaticano II, Sonderausgabe des „L'Osservatore della Domenica" vom 25. Januar 1966, S. 48, Spalte 1.

14. KB, a.a.O., Nr. 3a, S. 4.

15. Vgl. etwa: Xavier Rynne: Die zweite Reformation, Köln/Berlin 1964, S. 211.

16. KB, a.a.O., Nr. 4, S. 4.

17. 23 neue Konsultoren der Kommission für die Massenmedien, in: „KNA-Sonderdienst zum Zweiten Vatikanischen Konzil“, 25/26:1965, S. 12.

18. Xavier Rynne: Briefe aus dem Vatikan. Die zweite Sitzungsperiode des Zweiten Vatikanischen Konzils, Köln/Berlin 1964, S. 289.

19. Vergleichbar Generalisierend-Negatives bei: Leo Waltermann (Hrsg.): Konzil als Prozeß. Berichte im Westdeutschen Rundfunk über das Zweite Vatikanum. Eine Dokumentation, Köln 1966, S. 115-128.

20. Roegele, a.a.O., S. 322--324.

21. KB, a.a.O., Nr. 6a, S. 5.

22. Vgl. dazu: Josef Schmitz van Vorst: Kirche gestern - Kirche morgen. Aufzeichnungen 1962-1966, Stuttgart 1966, S. 288; Heinz Linnerz: Das Konzil hat gesprochen. Themen, Texte, Tendenzen, Kevelaer 1966, S. 212-219, hier S. 212; Manfred Plate: Weltereignis Konzil. Darstellung, Sinn, Ergebnis, Freiburg 1966, S. 162; Johann Christoph 
Hampe: Ende der Gegenreformation? Das Konzil. Dokumente und Deutung, Stuttgart/Berlin/Mainz 1964, S. 129-132; Joseph Ratzinger: Das Konzil auf dem Weg. Ruickblick auf die zweire Sitzungsperiode, Köln 1964, S. 72 f. - Eine besonders positive Deutung des Dekrets gibt John W. Mole: The Communications Decree of the Second Vatican Council, Charter of the communications apostolate, in: "Social Justice Review“, St. Louis Mo., 59:1966, S. 274-350.

23. Vgl.: Manfred Plate, a.a.O., S. 317.

24. Stanley I. Stuber: Der Wandel der Presse in der kirchlichen Erneuerung, Referat vor dem 8. Weltkongreß der Katholischen Weltunion der Presse (UCIP) vom 2. bis 6. Juli 1968 in Berlin, hekt., 9 Seiten. - Vgl. auch: Bericht in CS 1:1968, Heft 4, S. 336; Karl Rahner und Herbert Vorgrimler: Kleines Konzilskompendium, Freiburg 1966, S. 92.

25. KB, a.a.O., S. 7. - Vgl. auch: Werner Hamerski: Reden Papst Pius' XII. zu Fragen der Publizistik, in: „Publizistik“, Bremen, 8:1963, S. 611-631; - Radio Vaticana (Hrsg.): Documenti Pontifici sulla Stampa (1878-1963), Vatican o. J. (1964); Ders.: Documenti Pontifici sulla Radio e sulla Televisione (1929-1962), Vatican o. J. (1962).

26. Publiziert in: Herder-Korrespondenz, Freiburg, XVIII:1964, S. 498-501.

27. KB, a.a.O., Nr. II $1 \mathrm{a} / \mathrm{b}, \mathrm{S} .8$.

28. Herder-Korrespondenz, a.a.O., S. 500.

29. Der Begriff des „Sozialen“ birgt im deutschen Sprachgebrauch ein Dilemma, Er ist nicht, wie etwa im angelsächsischen Bereich, partnerschattlich-horizontal gerichtet, sondern auch vertikal und hat infolgedessen einen qualifizierend-caritativen Beiklang. Siehe dazu: Ralf Dahrendorf: Gesellschaft und Demokratie in Deutschland, München 1965 , S. 86.

30. Vgl. dazu: Dario Composta: Genesi storica del decreto, in: Enrico Baragli u. a. m. (Hrsg.): Gli Strumenti della Communicazione Sociale, Bd. 10 der "Collana Magistero Conclliare", Turin 1967, S. 19 ff.; Ebd.: Enrico Baragli: Sguardo d'insieme del decreto: nova et vetera, Kap. II: Valore di una nuova terminologia, S. 50-69.

31. Vgl. die ausführliche Kritik in KB, a.a.O., Nr. III, 1/2, S. 10.

31a. Ober die Abneigung der Konzilsväter gegenüber einer soldhen Deutung berichtet Konzilsperitus Johannes Hirschmann: Die bisherige Arbeit des Konzils. Ein schematischer Uberblick, in: Karl Forster (Hrsg.): Das Zweite Vatikanische Konzil, Würzburg 1963, S. 95-114, hier S. 103.

32. Roegele, a.a.O., S. 317.

33. Karl Rahner und Herbert Vorgrimler: Kleines Theologisches Wörterbuch, Freiburg 1961, Stichwort "Kirche", S. 199.

34. Vgl. auch: Eugen Biser: Kraft und Ohnmacht des Wortes, in: „Rundfunk und Fernsehen", Hamburg, 8:1960, S. $127 \mathrm{ff}$.

35. Am Institut für Publizistik der Universität Münster gehört „Religionspublizistik“ seit Jahren zu den bevorzugten Forschungsgebieten. Vgl. etwa: Henk Prakke und Gerhard E. Stoll: Entwurf zu einer Religionspublizistik, in: "Communicatio Socialis", $1: 1968$, S. 34-37; sowie die Definition dieses Wissenschaftsbereichs von Henk Prakke im Vorwort zu Franz Josef Eilers: Christliche Publizistik in Afrika. Eine erste Erkundung, Steyl 1964, S. 8.

36. KB, a.a.O., Nr. III 2, S. 10.

37. Vgl. dazu: Peter Pleyer: Der Kommunikationsprozeß als Dialog, in: „Katholische FilmKorrespondenz", Beilage zu Nr. 9/10 vom 15. Juni 1966, 6 Seiten, hier S. 3. - Ferner: Walter Hagemann: Grundzüge der Publizistik, Münster 21966, S. 47-49.

38. Roegele, a.a.O., S. 317.

39. Michael Schmolke: Fragen zum Ort von Gesinnungskräften im publizistischen Funktionieren, in: "Communicatio Socialis", 1:1968, S. 109-116, hier S. 111. Dort finden sich auch (S. 115) die wichtigsten Literaturhinweise zu diesem Themenbercich.

40. Siehe vor allem: Henk Prakke, Franz W. Dröge, Winfried B. Lerg, Michael Schmolke: Kommunikation der Gesellschaft. Einführung in die funktionale Publizistik, Münster 1968, S. 61 f. - Vgl. auch: Walter Hagemann, a.a.O., S. 55 f.

41. Prakke, Dröge, Lerg, Schmolke; a.a.O., S. 135.

42. Die Wissenschaft spricht nüchterner von Sozialisation als sozialer Orientierung in der Mitwelt, vgl. dazu: Prakke u. a. m., a.a.O., S. 160.

43. Roegele, a.a.O., S. 323.

44. Ansprache Pius' XII. "L'importance de la presse" vom 17. 2. 1950, in: AAS, 42:1950, S. 256; vgl. auch: Werner Hamerski, a.a.O., S. 615.

45. KB, a.a.O., Nr. III 10, S. 14. 
46. Gunnel Vallquist: „Das Zweite Vatikanische Konzil“, Nürnberg 1966, S. 146 f.

47. Vgl. etwa: Karlheinz Peschke: Naturrecht in der Kontroverse, Salzburg 1967, 191 S.

48. Zum erstenmal wirft diese Frage Michael Schmolke, a.a.O., S. 111, auf.

49. Gary Mac Eoin: Was geschah in Rom? Paderborn 1967, S. 154.

50. Roegele, a.a.O., S. 320.

51. Vgl. ctwa: Dic gute und die schlechte Presse, in: "Historisch-politische Blätter für das katholische Deutschland", München, 5. Bd. 1840, Tcil XIV, S. 152-169.

52. Vgl. in dieser Ausgabe von CS: Seite 5-12; Seite 41-44.

53. Vgl. etwa: Walter Hagemann, a.a.O., S. 76.

54. So im Februar 1964 im Institut für Publizistil der Universität Münster/Westf.

\section{SUM M A R Y}

In the postconciliar literature, especially in the commentaries on Council documents, the decree "Inter Mirifica" on the instruments of Social Communications was until now somehow neglected. Still waiting for the coming Pastoral instruction, the few commentaries published have therefore a special value. In Germany this is particularly true for a "scientific" commentary on "Inter Mirifica“ in a three-volume collection of Council documents with comments („Das Zweite Vatikanische Konzil. Konstitutionen, Dekrete und Erklärungen", Freiburg 1966; Here: volume I, page 111-135) was published in connection with the well-known "Lexikon für Theologie und Kirche". This commentary has considerable shortcomings. So there is e. g. no indication of literature or other sources although great parts of the introduction are nearly word for word taken from a study of Otto $B$. Roegele on the subject (O. B. Rocgele, Das Konzilsdekret „Ober die Werkzeuge der sozialen Kommunikation" in: "Publizistik", Bremen, 9:1964, page 305-347). The introduction on the history of the origin and rise of the decree is one-sided, negative and the commentary itself eclectical. Secondary things are overestimated and important texts are only commented on in between. Often the author restricts himself on a somehow changed repetition of the text of the decree itself. Where he is deploring scientific shortcomings, he doesn't himself have the knowledge and the terminology to describe these. Even his demand to correct the translation approved by the bishops the author can't fulfill. - The very same commentary is even published in English: H. Vorgrimler (Editor), Commentary on the Documents of Vatican II (4 volumes), New York and London $1967 \mathrm{f}$.

\section{RESUMEN}

En la literatura postconciliar, sobre todo en la interpretación de los textos del Concilio, no se ha dado todavía la importancia que merece al Decreto sobre los Medios de Comunicación Social, „Inter Mirifica“. Mientras se espera la publicación de la Instrucción Pastoral de este Decreto, sus comentarios tienen una importancia particular. Esto sucede sobre todo en Alemania, donde ha aparecido una explicación "científica" de "Inter Mirifica" en una edición explicativa del Concilio, tres tomos, publicada como Apéndice en el conocido "Lexikon für Theologie und Kirche“, bajo el título: „Das Zweite Vatikanische Konzil. Konstitutionen, Dekrete und Erklärungen" (Teil I, Freiburg 1966, S. 111-135). Desgraciadamente, esta publicación muestra vacíos considerables. No ofrece datos bibliográficos ni citas de autores, aunque, por ejemplo, gran parte de la Introducción ha sido tomada casi literalmente del trabajo previo de Otto B. Roegele (Das Konzilsdekret ,Ueber die Werkzeuge der sozialen Kommunikation', publicado en „Publizistik“, Bremen $9: 1964$, S. 305-347). La introducción sobre el origen del Decreto es unilateralmente negativa, el comentario propiamente dicho es ecléptico. Se acentua exageradamente lo secundario, mientras que lo importante se interpreta superficialmente. Frecuentemente, el autor se limita a una reproducción indirecta del texto conciliar. Allí donde se lamenta de los fallos científicos de "Inter Mirifica“, no basta su conocimiento y terminología propia para exponer exactamente esos fallos. Aunque el autor se gloría de haber revisado la edición alemana del Decreto, aprobada por el episcopado, no consigue estar a la altura de su reivindicación. 